\title{
The role of external fixation in the treatment of humeral shaft fractures: A retrospective case study review on 85 humeral fractures
}

\author{
Michelangelo Scaglione, Luca Fabbri, Dario Dell' Omo, Andrea Goffi, Giulio Guido
}

\begin{abstract}
There is no consensus among surgeons on the treatment for humeral fractures: the best it is still a matter of some debate. The aim of our work was to demonstrate that external fixation may be considered a valid method not only in emergencies but also for the definitive treatment of such fractures. We perform a retrospective case study review on 85 humeral fractures, 62 shaft fractures, and 23 extrarticular distal third fractures treated with external fixation. Clinical (Disabilities of the Arm, Shoulder and Hand (DASH) score and SF-36) and radiographic follow-up lasted on average 30 months (minimum 12 to maximum 36). Complete healing of fractures was achieved in $97.6 \%$ of cases ( 83 patients), with an average consolidation time of about 12 weeks (83.2 days). One case of delayed union and one case of refracture were encountered. Eighty-one patients demonstrated SF-36 scores at or above the national average and an average DASH score of 8.9. External fixation of humeral shaft fractures is considered a valid treatment method as it provides good results in terms of stability of reduction, tolerability, healing times, and functional recovery.
\end{abstract}

\section{INTRODUCTION}

Humeral shaft fractures represent approximately $2-3 \%$ of all fractures, having an average incidence of 14 out of 100,000 [1], [2], [3].

They present a bimodal peak incidence: they are more frequent in males under 50 years of age and in females over 70 .

In the case of males, the causative event is generally high-energy trauma due to road accidents, sports injuries, or falls from a considerable height.

On the other hand, in females, in addition to high-energy trauma, low-energy impacts such as a fall at home from a modest height often precipitate such fractures due to a clinical condition of osteoporosis [1], [4].

The most frequent and dangerous complication of humeral shaft fractures is represented by damage to the radial nerve, which runs along the rear surface of the bone in the spiral groove of the humerus. This lesion is present in $11.8 \%$ of all cases (15.2\% of all shaft fractures) and most frequently associated with spiral Holstein-Lewis fractures [5].

The nerve may be bruised or stretched by the fracture fragments, or may even tear. In the latter case, the onset of paralysis is abrupt and presents as a deficit in extension of the finger (falling hand) and wrist, with hypo-anaesthesia of the first and second fingers and the first and second metacarpus on the back of the hand.

Partial functional recovery may take several months and is usually complete within 2 years, so progress should be monitored with electromyograph studies. 
In most closed fractures, up to $100 \%$ for some authors, radial nerve recovery is complete and it can last up to 6 months. Surgical revision of the nerve is necessary only if functional recovery has not yet begun after 6 months from the traumatic event [6], [7].

Other complications that may occur in humeral shaft fractures are nonunion and brachial artery injury.

The appearance of nonunion is extremely variable, from $2 \%$ to $33 \%$ of humerus fractures, its occurrence depending on many concomitant causes and factors[8], [9].

In humeral fractures, nonunion is defined as the radiographic detection of delayed consolidation of the fracture 6/8 months after treatment [10], [11].

The main cause is instability of the fracture (i.e., the presence of abnormal movements at the fracture site) due to inadequate treatment and poor reduction. Most fractures exhibiting nonunion are revealed to have been treated with conservative methods [12].

According to several authors, obesity, defined as body mass index (BMI)> 30, is a contributory cause factor in 35-37\% of nonunions. Other predisposing risk factors, in addition to the already mentioned instability of the reduction, are the daily use of tobacco (38-53\%), cardiovascular disease (37\%), metabolic bone disease (32\%), and polytrauma with multiple fractures of long bones [13], [14].

Another cause of nonunion is the exposure of the fracture, which causes the loss of fracture hematoma, compromising the beginning of the consolidation process. In addition, exposure can also lead to bone fracture infection, resulting in non-union [10], [11].

Finally, bone necrosis due to poor blood supply is another condition favouring evolution towards nonunion.

Injury of the brachial artery is a rare but dreaded complication that requires urgent treatment.

In recent years, the most commonly used treatment methods for humeral shaft lesions have been conservative, with casts and braces, and surgical with internal fixation, intramedullary nailing (IMN), and external fixation.

Our experience with external fixation devices has allowed us to assess how the use of the fixators produced clinically and radiographically similar results to other more invasive methods, while affording the advantages of speed and low invasiveness.

In the following we review the cases of humeral shaft fracture treated in our clinic between 2001 and 2010 with the aim of determining whether the use of external fixators should be considered a valid method, not only in the event of an emergency but also for the definitive treatment of such fractures.

\section{MATERIALS AND METHODS}

We treated 85 patients (63 males and 22 females) with external fixation out of a total of 270 humeral fractures who underwent surgery. The mean follow-up was 30 months (minimum 12 to maximum 36). 
The patients' average age was 43.9 years (minimum 10 to maximum 86 ).

Most of the fractures resulted from high-energy trauma in young-adult males.

In nine cases the patients had undergone polytrauma, with other skeletal segment lesions. The fractures were graded according to the AO Foundation and Orthopaedic Trauma Association (AO/OTA) classification.

Applying this classification to our series, we found:

12-A1: 12; 12-A2: 12; 12-A3:4; 12-B1:18; 12-B2:4; 12-B3:4; 12-C1:3; 12-C2:2; 12-C3:3; 13-A1: none; $13-$ A2:19; and 13-A3:4

All patients had a soft tissue lesion greater than or equal to grade 2 Tscherne.

There were also four exposed fractures.

We used the Stryker Hoffmann type II external fixator, which is a modular fixator consisting of aluminium and carbon fibres and aluminium bars.

The operative technique consists of placing the patient supine on the operating table with the affected arm abducted at $45-60^{\circ}$ and the elbow flexed at $90^{\circ}$.

Anaesthesia is generally locoregional, with continuous interscalene brachial plexus block. This technique enables immediate passive mobilisation of the operated segment thanks to the possibility of prolonging the analgesic effect in the postoperative period.

The screws are self-tapping with a diameter of 4 or $5 \mathrm{~mm}$ and are always inserted manually, usually two proximal and two distal to the fracture site, depending on the complexity of the fracture. Some interfragmentary screws can be used to better stabilise the fracture.

For the insertion points, we follow the technique and mapping described by Professor Bianchi Maiocchi [15] to fix all the screws on the lateral humerus.

One screw is inserted in a position just proximal to the olecranon fossa under fluoroscopic guidance: in order to avoid the ulnar nerve, we proceed with lateral-to-medial insertion of a Kirschner wire (K-wire), slightly tilted in the posterior-anterior direction in a selected area of the lateral cortex. Then we remove the K-wire and use its entrance hole on the lateral cortex as a guide for the first screw.

The second screw is fixed on the same plane as the first, keeping the elbow flexed and the arm abducted. This will slacken and shift the radial nerve forward. The area chosen for insertion is a safe zone $8.5 \mathrm{~cm}$ proximal to the epicondyle. To improve security, we use the anchor positions $1-4$ or $2-4$ of the clamp.

The proximal screws are inserted into the lateral humerus, proximal to the "V" of the deltoid muscle, accessing the bone via a blunt dissection through the muscle fibres of the deltoid.

We proceed to the installation of the connecting bars, usually two, and reduction of the fracture under fluoroscopic guidance.

Then we close the system and stabilise it with a crossbar to increase the stability of the implant.

The use of two proximal and two distal screws and a "long" implant lends proper flexibility and stability to the fracture. Stability is further increased for the first 30 days by the crossbar connection. 
As a final step, any intermediate fragments are set. In cases where it was necessary to fix a third fragment (medial, butterfly), we follow AO techniques for the stabilisation of small fragments: a 5-mm hole is effected on the lateral cortex and then a 4-mm-diameter screw is used to pierce the medial cortical fragment to enable it to be pulled through, so as to improve fracture reduction and implant stability. [fig. 1]

In the postoperative period, antibiotic prophylaxis is continued for 5 days, and patients are subjected to early mobilisation of the operated arm.

The screws should be medicated every other day with sterile saline solution or hydrogen peroxide.

Patients were followed up with clinical checks every 15 days in a specialised ward in order to assess the condition of the skin around the screws, and a clinical and radiographic exam every 30 days to assess the progress of fracture consolidation. After 30 days, dynamisation of the fixation system was performed removing the crossbar connection. The external fixator was removed through a daysurgery hospitalisation after complete healing of the fracture.

Patients underwent further clinical and radiographical follow-up, which lasted for an average of 2.5 years.

The results were evaluated from both a clinical and radiographic point of view, considering the average consolidation time, and the onset of any nonunion, refracture, and/or angular defects.

The most widely used health international questionnaire the Short Form Health Survey (SF-36) was used to standardise the results in terms of functional limitations to patients.

The SF-36 offers the advantages of being fast (the interview takes only a few minutes) and easily reproducible. It is a multidimensional questionnaire consisting of 36 questions that explore eight health domains: AF (physical activity), RP (role limitations due to physical health), RE (role limitations due to emotional state), BP (bodily pain), GH (general health perception), VT (vitality), SF (social activities), MH (mental health), and a single question on the patient's perceived change in health status.

The results were standardised by sex and age according to the average values in the population.

The results are reported in terms of two indices: physical health $(\mathrm{PH})$ and mental health $(\mathrm{MH})$, which are easily comparable with statistics on the general population.

We also evaluated our patients with DASH (Disabilities of the Arm, Shoulder and Hand), a questionnaire that measures functioning and symptoms in patients with any musculoskeletal upper limb impairment.

A score of 0 indicates a state of optimal health, while a score of 100 indicates total disability of the upper limb. This tool provides greater specificity for lesions of the upper limb than the SF-36 and is therefore very useful for evaluating the effective recovery of arm function in polytrauma patients.

\section{RESULTS}

The 83 fractures $(97.6 \%)$ in our series were consolidated in a mean time of 83.2 days (12 weeks). 
There was one delayed union (1.2\%), one refracture (1.2\%), and five angular defects between $10^{\circ}$ and $20^{\circ}(7.2 \%)$. We had one case of neuro-apraxia of the radial nerve, which spontaneously and completely resolved after 2 months. No iatrogenic radial nerve injury occurred in consequence to insertion of the external fixator.

Two superficial infections (2.4\%) and one suspected deep infection (1.2\%) occurred and were resolved simply by removing the external fixator in addition to antibiotic therapy.

By standardizing the data for our clinical series using the SF-36, we obtained:

- eighty-one good results, in which the patient did not report any restrictions on their work or normal social activities (SF36 scores at or above the national average) and

- four poor results with scores below the national SF36 average. In these cases, the patient had to reduce or change their normal daily activities.

Applying the DASH score to our series yielded excellent results, with an average score of 8.9.

\section{DISCUSSION}

Even today, there is still no universally agreed-upon method of treatment for humeral shaft fractures.

Many methods of treatment, both surgical and conservative, have been proposed over the years by various authors.

Simple fractures without bone exposure have long been treated with nonsurgical methods. Such methods, the first to be implemented, are still considered valid, despite the disadvantages of requiring long healing times and being difficult to manage, especially in uncooperative patients.

Surgeon experience and newer studies assessing functional outcomes in nonoperative patients have challenged the belief that humeral shaft fractures uniformly do well without surgery [16].

The two primary methods of definitive operative fixation are IMN and compression plating [17], [18].

A recent meta-analysis of plate fixation versus IMN for humeral shaft fractures suggested that plating is superior to IMN for humeral shaft fractures [19]. All told, however, the plates are considered better than the nails, because they ensure similar results without the risk of compromising the function of the shoulder or elbow [20].

With modern implants and surgeons adept to their use, humeral union and functional outcomes have been shown to be the same between plates and nails [21].

Moreover, plate osteosynthesis is a rather invasive method. According to Zhiquan et al., reduced invasiveness can be achieved with the use of minimally invasive plate osteosynthesis (MIPO) [19]. 
The international literature contains very few articles on external fixators for treating humeral shaft fractures, and the only reported case studies regard severely exposed fractures (Gustilo-Anderson type 2-3).

External fixation does play a role and is increasingly used in polytrauma patient or combat setting for temporary stabilisation; however, its use for the definitive management of humeral shaft fractures is limited and not generally advised because of the concern for deep infection [22].

Additional indications for external fixation application include severe soft tissue injuries serious exposed bone fractures (Gustilo type 2-3), where there are severe soft tissue injuries $\left(2-3^{\circ}\right.$ Tscherne), vascular injuries requiring quick stabilisation before repair, and an unstable elbow joint after bony fixation [17],[23], [24], [25].

It also has the advantage of being a physiologically 'friendly' method that fully respects the fracture hematoma, thereby reducing the risk of eventually developing pseudarthrosis [17].

It is moreover very useful in achieving rapid stabilisation of a fracture in polytrauma patients for whom other diagnostic and therapeutic procedures must take priority (head injury and abdominal trauma).

External fixation has been adopted by our team as a definitive method for a number of reasons: ease of installation, speed of surgical execution, good stabilisation of the fracture, and maintenance of the fracture reduction over time.

External fixation is also less invasive than other methods and has no associated risk of injury to the rotator cuff or elbow joint.

The risk of radial nerve damage is also quite low. Clement et al. [21], [26] found a relative frequency of such lesions in four screws out of every 40 implanted in the humerus.

However, our series did not result in any injuries to the radial nerve from treatment with an external fixator. Such good results seem to stem from careful adherence to the surgical technique: the limb positioning, always flexed at $90^{\circ}$; insertion of the screws distally, in the safety zone $(8.5 \mathrm{~cm}$ from the epicondyle); and the reduction manoeuvre. Some authors even use external fixators in injuries of the radial nerve.

The patients' compliance to the external fixator was quite good. As reported by patients, our frame for the humerus is not bulky and allows almost all normal daily activities to be performed. [fig. 2]

Insertion of the screws on the deltoid muscle was well tolerated by patients, who reported no pain, functional limitations, infection, or other complications. Despite the amount of space occupied by the frame, external fixation is well tolerated, more than other district. In fact, the majority of patients start to move the shoulder and elbow the first few days after intervention and the range of motion normally is complete after 20-25 days.

The use of an external fixator requires close monitoring: the patient should be assessed every 15 days to check the screws and make a careful clinical evaluation to avoid the occurrence of complications such as infections.

The infections observed were all superficial and mainly due to the tension exerted on the skin by the screws and were treated with small incisions under local anaesthesia and oral antibiotic therapy. 
One feature that distinguishes external fixation from other treatment methods and which is very important for good fracture healing is the ability to provide stability without excessive rigidity.

The timing of the consolidation of the fractures treated with external fixation in this series (89.2 days) is in line with those of other methods: a bit longer than for intramedullary nails (82.9), though a bit shorter than for plate osteosynthesis (94.2) [27].

As for the poor results according to SF-36, these can be at least partly attributed to the associated lesions incurred by some polytrauma patients.

In cases of multiple trauma, because the SF-36 is a generic health status questionnaire, it is often difficult to determine how much of the resulting limitation is due to the humerus fracture and how much due to any associated injuries.

\section{CONCLUSION}

The treatment of humeral shaft fractures using an external fixator enables achieving similar results to more invasive surgical techniques. Indeed, it offers the combined advantages of a simple, physiological, and minimally invasive surgical technique, good patient tolerability, and good stability of the fracture reduction.

In the series presented herein good results were obtained in $94.5 \%$ of cases, with only one delayed union, mainly indicated in type $12 \mathrm{~B}$ and $\mathrm{A} 2$ fractures (A3 fractures require longer healing periods) and in $13 \mathrm{~A} 2, \mathrm{~A} 3$.

The further benefits of external reduction include the possibility of mobilizing the treated limb immediately and the excellent functional recovery obtainable from both the clinical and radiographic standpoints (demonstrable through suitable tests, and considering the patient's state of health).

In agreement with Ruland, we believe that external fixation is indicated also in fractures of the distal third of the humerus, in the treatment of bilateral fractures of the humerus and fractures with radial nerve injuries, as well as in the treatment of hypertrophic nonunion and infection. It is a minimally invasive technique that definitely provides maximum protection of the soft tissues and maintenance of the fracture hematoma to promote callus formation [28].

However, external fixation does suffer from some drawbacks, including greater staff exposure to radiation and the development of superficial infections. Therefore, strict monitoring of the patient in a specialised ward is in order.

We therefore believe that external fixation should be considered a valid treatment method for humeral shaft fracture, not only in emergencies but also for definitive reduction of such lesions. 


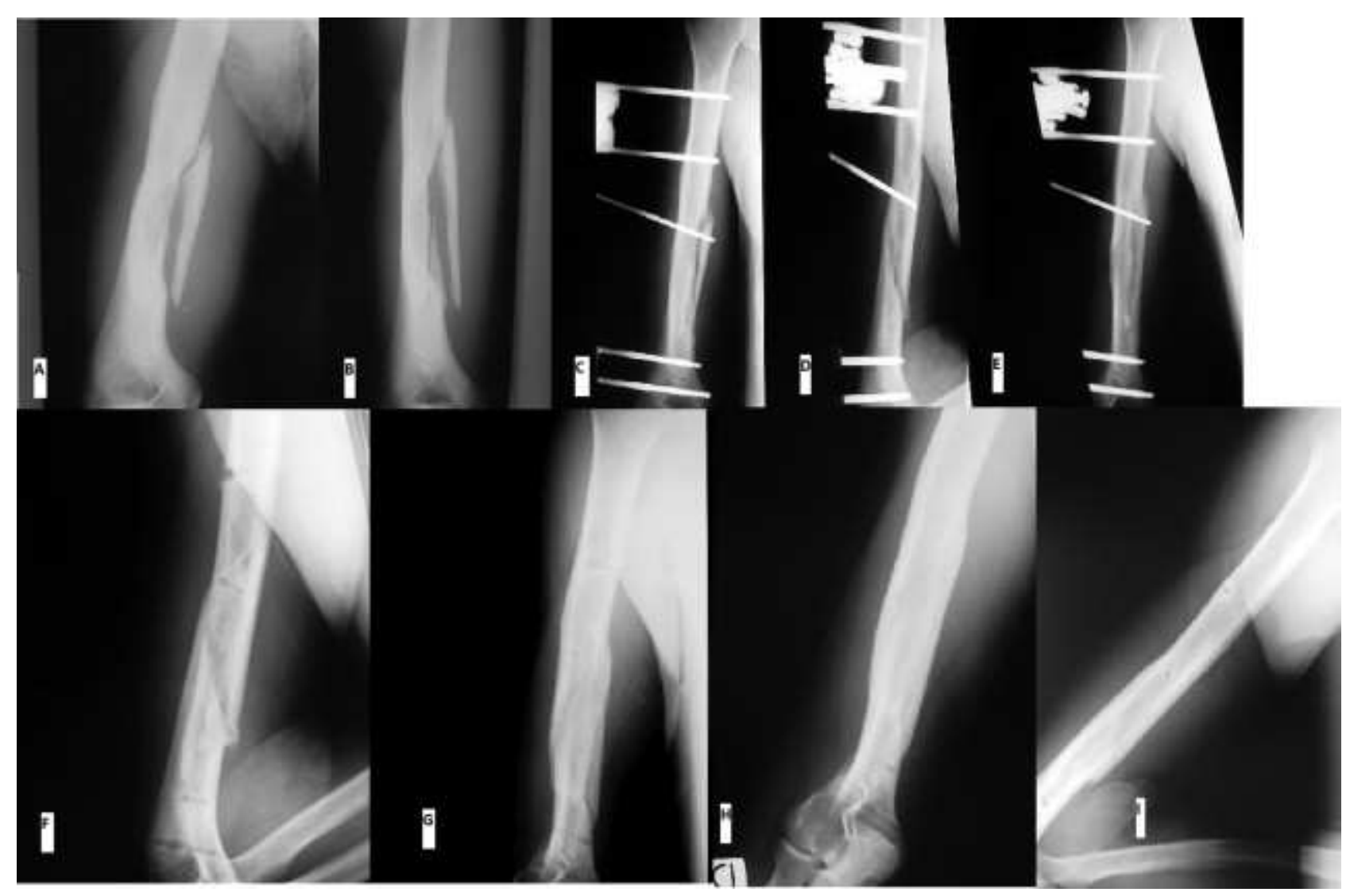

Fig. 1

Caucasian male, 55 yo: ((A) and (B)) X-ray at time of fracture. ((C) and (D)) X-ray 30 days after surgery. (E) $\mathrm{X}$-ray 60 days after surgery. $((\mathrm{F})$ and $(\mathrm{G}))$ The ExFix was removed after 75 days. $((\mathrm{H})$ and $(\mathrm{I})) \mathrm{X}$-ray 5 months after removal. 


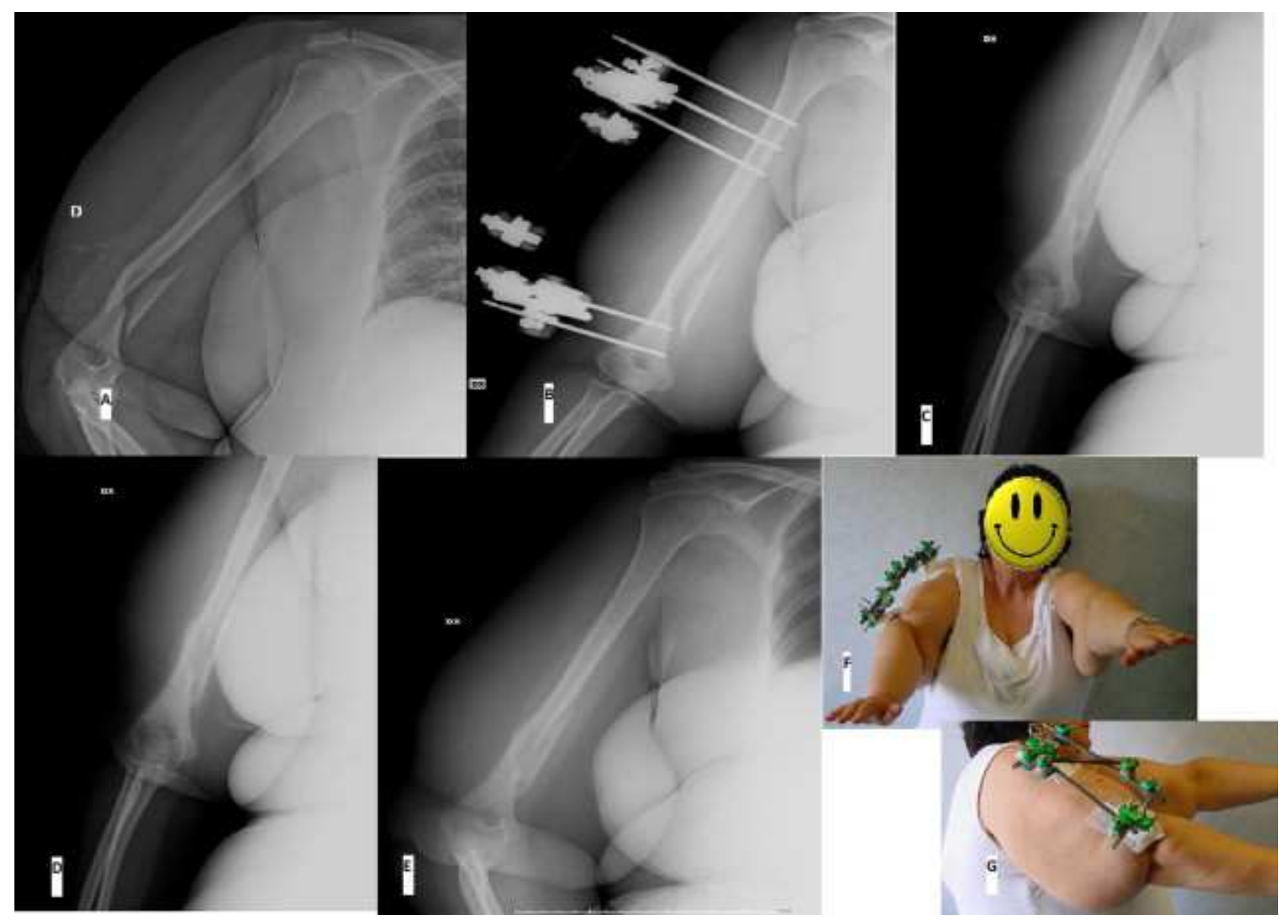

Fig. 2

Caucasian female 46 yo, obese (BMI 43): (A) X-ray at time of fracture. (B) X-ray 30 days after surgery. (C) The ExFix was removed after 70 days, X-ray after removal. ((D) and (E)) X-ray 4 months after removal. ((F) and $(\mathrm{G}))$ The external fixator was well tolerated despite the patient's weight.

\section{REFERENCES}

[1]. Ekholm R, Adami J, Tidermark J, Hansson K, Törnkvist H, Ponzer S. Fractures of the shaft of the humerus. an epidemiological study of 401 fractures. J Bone Jt Surg Ser B [Internet].

2006;88(11):1469-73

[2]. McKee MD. Fractures of the shaft of the humerus. Rockwood and Green's Fractures in Adults [Internet]. 2006;1:1117-59

[3]. Tsai C-, Fong Y-, Chen Y-, Hsu C-, Chang C-, Hsu H-. The epidemiology of traumatic humeral shaft fractures in taiwan. Int Orthop [Internet]. 2009;33(2):463-7

[4]. Tytherleigh-Strong G, Walls N, McQueen MM. The epidemiology of humeral shaft fractures. J Bone Jt Surg Ser B [Internet]. 1998;80(2):249-53

[5]. Shao YC, Harwood P, Grotz MRW, Limb D, Giannoudis PV. Radial nerve palsy associated with fractures of the shaft of the humerus. A systematic review. J Bone Jt Surg Ser B [Internet]. 2005;87(12):1647-52. 
[6]. De Franco MJ, Lawton JN. Radial nerve injuries associated with humeral fractures. J Hand Surg (USA) [Internet]. 2006;31(4):655-63

[7]. Ekholm R, Ponzer S, Törnkvist H, Adami J, Tidermark J. Primary radial nerve palsy in patients with acute humeral shaft fractures. J Orthop Trauma [Internet]. 2008;22(6):408-14

[8]. Ekholm R, Tidermark J, Törnkvist H, Adami J, Ponzer S. Outcome after closed functional treatment of humeral shaft fractures. J Orthop Trauma [Internet]. 2006;20(9):591-6

[9]. Livani B, Belangero W, Medina G, Pimenta C, Zogaib R, Mongon M. Anterior plating as a surgical alternative in the treatment of humeral shaft non-union. Int Orthop [Internet].

2010;34(7):1025-31

[10]. Rosen H. The treatment of nonunions and pseudarthroses of the humeral shaft. Orthop Clin North Am [Internet]. 1990;21(4):725-42

[11]. Atalar AC, Kocaoglu M, Demirhan M, Bilsel K, Eralp L. Comparison of three different treatment modalities in the management of humeral shaft nonunions (plates, unilateral, and circular external fixators). J Orthop Trauma [Internet]. 2008;22(4):248-57

[12]. Rodríguez-Merch Á N EC. Compression plating versus hackethal nailing in closed humeral shaft fractures failing nonoperative reduction. J Orthop Trauma [Internet]. 1995;9(3):194-7

[13]. Green E, Lubahn JD, Evans J. Risk factors, treatment, and outcomes associated with nonunion of the midshaft humerus fracture. J Surg Orthop Adv [Internet]. 2005;14(2):64-72

[14]. Decomas A, Kaye J. Risk factors associated with failure of treatment of humeral diaphyseal fractures after functional bracing. J La State Med Soc [Internet]. 2010;162(1):33-5

[15]. Catagni MA. Atlas for the Insertion of Transosseous Wires and Half-Pins Ilizarov Method [Internet]

[16]. Spiguel AR, Steffner RJ. Humeral shaft fractures. Curr Rev Musculoskelet Med [Internet]. 2012;5(3):177-83

[17]. Wang X, Chen Z, Shao Y, Ma Y, Fu D, Xia Q. A meta-analysis of plate fixation versus intramedullary nailing for humeral shaft fractures. J Ortop Sci [Internet]. 2013;18(3):388-97

[18]. Hems TEJ, Bhullar TPS. Interlocking nailing of humeral shaft fractures: The oxford experience 1991 to 1994. Injury [Internet]. 1996;27(7):485-9

[19]. Zhiquan A, Bingfang Z, Yeming W, Chi Z, Peiyan H. Minimally invasive plating osteosynthesis (MIPO) of middle and distal third humeral shaft fractures. J Orthop Trauma [Internet]. 2007;21(9):628-33

[20]. Chapman JR, Henley MB, Agel J, Benca PJ. Randomized prospective study of humeral shaft fracture fixation: Intramedullary nails versus plates. J Orthop Trauma [Internet]. 2000;14(3):162-6

[21]. Heineman DJ, Poolman RW, Nork Sean SE, Ponsen K-, Bhandari M. Plate fixation or intramedullary fixation of humeral shaft fractures: An updated meta-analysis. Acta Orthop [Internet]. 2010;81(2):218-25 
[22]. Dougherty PJ, Silverton C, Yeni Y, Tashman S, Weir R. Conversion from temporary external fixation to definitive fixation: Shaft fractures. J Am Acad Orthop Surg [Internet].

2006;14(10):S124-7

[23]. Marsh JL, Mahoney CR, Steinbronn D. External fixation of open humerus fractures. Iowa Orthop J [Internet]. 1999;19:35-42

[24]. Mostafavi HR, Tornetta III P. Open fractures of the humerus treated with external fixation. CLIN ORTHOP RELAT RES [Internet]. 1997(337):187-97.

[25]. Zinman C, Norman D, Hamoud K, Reis ND. External fixation for severe open fractures of the humerus caused by missiles. J Orthop Trauma [Internet]. 1997;11(7):536-9

[26]. Clement H, Pichler W, Tesch NP, Heidari N, Grechenig W. Anatomical basis of the risk of radial nerve injury related to the technique of external fixation applied to the distal humerus. Surg Radiol Anat [Internet]. 2010;32(3):221-4

[27]. Cheng H-, Lin J. Prospective randomized comparative study of antegrade and retrograde locked nailing for middle humeral shaft fracture. J Trauma Inj Infect Crit Care [Internet]. 2008;65(1):94-102

[28]. Ruland WO. Is there a place for external fixation in humeral shaft fractures? Injury [Internet]. 2000;31(SUPPL. 1):27-34 